\title{
A study protocol for a randomised controlled trial of ice to reduce the pain of immunisation
}

\author{
The ICE trial
}

Yashodha Ediriweera, Jennifer Banks, Leanne M Hall, Clare Heal

\section{Background and objective \\ Vaccine injections commonly cause iatrogenic pain. Ice may reduce pain. This is a study protocol for a prospective randomised controlled trial aiming to investigate the efficacy of ice packs to decrease vaccine-related pain in adults.}

\section{Methods}

The intervention group $(n=45)$ will receive ice packs $\left(0^{\circ} \mathrm{C}\right)$ and the control group $(n=45)$ receive placebo cold packs $\left(10^{\circ} \mathrm{C}\right)$ on the injection site for 30 seconds prior to vaccination.

\section{Results}

The primary outcomes will test the hypothesis that applying an ice pack will significantly reduce pain of vaccination when compared with a cold pack. The primary dependent variable will be pain score recorded on a visual analogue scale. Secondary outcomes aim to assess potential adverse reactions. The main analysis of data will follow the intentionto-treat principle. The primary dependent variable will be compared using an independent t-test or a Mann-Whitney $\mathrm{U}$ test. Multivariable logistic regression analysis may be used.

\section{Discussion}

The trial may guide general practitioners in the use of ice packs to reduce injection-related pain in adults.
VACCINE INJECTIONS are a common cause of iatrogenic pain in childhood and a cause of anxiety in adults. ${ }^{1,2}$ A 2003 survey reported $21-90 \%$ of adults experienced anxiety related to pain associated with needle-based procedures. ${ }^{3}$ In severe cases, this leads to injection phobia. ${ }^{4}$ Vaccinationsite pain is now a recognised adverse effect, and adequate pain management strategies should be incorporated into every vaccination. ${ }^{5}$

Multiple theories are used to explain the physiological mechanisms for the analgesic effects of cold temperature. The gate control theory of pain by Melzack and Wall in 1965 theorised that noxious inputs relayed by small, myelinated $A \delta$ fibres and unmyelinated $\mathrm{C}$ fibres are inhibited by non-noxious stimuli concurrently conducted by $\mathrm{A} \beta$ fibres to the dorsal horn. Consequently, the intensity of the ascending pain stimuli is decreased. ${ }^{6}$ Kunesch et al showed that effects of skin cooling were more pronounced on $\mathrm{C}$ fibres when compared with A fibres, leading to reduced autonomic response and pain sensation. ${ }^{7}$ Another theory is activation of the analgesic descending pathway stimulated by cold, which produces opiate-like peptides. ${ }^{8}$ In general, ice packs $\left(<5^{\circ} \mathrm{C}\right)$ are known to reduce the temperature of skin and tissues up $2 \mathrm{~cm}$ deep and rapidly induce localised skin analgesia..$^{9,10}$ A previous study showed that a 30-second application of ice reduces pain during adult tetanus vaccinations. ${ }^{11} \mathrm{~A}$ cold pack of $10^{\circ} \mathrm{C}$ requires a minimum 20 -minute application to achieve a similar decrease in skin temperatures and is limited in its ability to provide rapid skin analgesia. ${ }^{10}$

Literature on the use of cooling for injection-related pain shows conflicting results. ${ }^{12}$ Vapocoolant spray has been shown to be effective in adult and paediatric populations, while in limited paediatric studies ice was not effective. ${ }^{1,11,13}$ Only one randomised controlled trial (RCT) has investigated the use of ice to reduce vaccine-related pain in adults, showing a reduction in pain when compared with a no-intervention control group. ${ }^{11}$ As summarised by Hall et al, ${ }^{12}$ there may be many reasons why previous studies have shown mixed results, including age of participants, outcome measures used, comparator groups that include other interventions, administration of different types and/or number of vaccines, and variable administration of chosen cooling techniques. A lack of RCTs that investigate ice as a means of mitigating pain during vaccination, especially in adults, leaves a gap in the evidence for or against the use of this modality; hence, further research is needed.

\section{Hypothesis and objectives}

The primary aim of this RCT is to investigate the effectiveness of application 
of an ice pack at the site of injection for 30 seconds immediately prior to influenza vaccination in reducing injection-related pain. In addition, the aim is to observe any potential adverse reactions to either the ice pack or influenza vaccines.

It is hypothesised that ice will reduce vaccination-related pain levels more than a placebo cold pack.

\section{Methods}

\section{Study design and trial registration}

The study protocol for this multicentre, double-blinded prospective RCT was registered with the Australian New Zealand Clinical Trials Registry (ANZCTR) - ACTRN12621000064808.

The 'Standard Protocol Items:

Recommendations for Interventional Trials (SPIRIT)' guideline was used to report the protocol. ${ }^{14}$

\section{Setting and sampling participants}

The study will be conducted in two medical student immunisation clinics affiliated with James Cook University Medical Student Association (JCUMSA) in Mackay and Townsville, Queensland. Medical students presenting to Mackay City Medical Practice and JCU Health for influenza vaccination clinics will be eligible to participate.

\section{Eligibility criteria}

Patients over the age of 18 years undergoing influenza vaccination at participating vaccination clinics during the study period who have the capacity to provide informed consent, are able to return for the assessment of the vaccination reaction and have no history of reactions to ice are eligible to participate. Patients will be excluded if they have known histories of cold anaphylaxis, serious immunisation reactions or allergy to influenza vaccines.

\section{Randomisation and blinding}

The study will be double blinded. Randomisation will be performed by the primary investigator at the level of the patient with an allocation ratio of 1:1. A computer-generated random number table will be used. Allocation concealment will be attained by using sealed, numbered, opaque envelopes. ${ }^{15}$ Doctors and nurses involved in the assessment or treatment of patients will have no role in the assignment process. Further, ice packs and cold packs will be stored in two coolers labelled A and B, respectively, to blind the doctor administering vaccines.

\section{Interventions: Ice packs $\left(0^{\circ} \mathrm{C}\right)$ versus cold packs $\left(10^{\circ} \mathrm{C}\right)$}

Participants will have a $4 \times 6 \mathrm{~cm}$ ice pack $\left(0^{\circ} \mathrm{C}\right.$; intervention group) or $4 \times 6 \mathrm{~cm}$ cold pack $\left(10^{\circ} \mathrm{C}\right.$; control group) applied to the site of injection for 30 seconds prior to their influenza vaccination.

\section{Vaccination protocol}

A vaccination protocol, modelled on The Royal Australian College of General Practitioners' (RACGP's) immunisation and influenza prevention guidelines, was developed in consultation with participating doctors and nurses. ${ }^{16,17}$ One doctor will apply the ice or cold packs while a second doctor will administer influenza vaccines immediately following application. Pre-filled influenza vaccine syringes from QML pathology with standard identical needle size will be used. Post-immunisation processes will be identical across all sites, with all patients receiving a standard set of verbal and written post-immunisation instructions.

\section{Visual analogue scale and sample size calculation}

While there is no gold standard of assessing acute pain severity, the visual analogue scale (VAS) is a widely used pain scale because of its simplicity and adaptability to a broad range of clinical settings and populations. ${ }^{18}$ The researchers opted for a VAS of $100 \mathrm{~mm}$ for measuring post-immunisation pain, as has been used in previous studies that have investigated adult vaccination pain. ${ }^{13,11,19}$ The minimum clinically significant difference in VAS scores ranges between $13 \mathrm{~mm}$ and $17 \mathrm{~mm}$, thus a midpoint of $15 \mathrm{~mm}$ was chosen for the present study. ${ }^{20}$ VAS has a standard deviation of $26 \mathrm{~mm} .^{21}$ A sample size of 45 per group is required to achieve $80 \%$ power with $P=0.05$.

\section{Data collection}

Baseline demographic data will be collected, including age, sex, ethnicity and past medical history. The primary outcome measure is the difference in the level of pain on VAS between the two study groups at the time of vaccination. Participants will be asked to record their immediate post-immunisation pain on a pre-printed $100 \mathrm{~mm}$ VAS and return the form to a collection box placed outside the doctor's room before leaving the premises (Figure 1).

The secondary study outcome includes adverse reactions to the vaccination process, manifesting as any one of anaphylaxis, skin irritation, redness or contact dermatitis. Participants will be advised to assess the injection site 24 hours post vaccination, complete a self-assessment form and return it to the clinic within 48 hours of vaccination

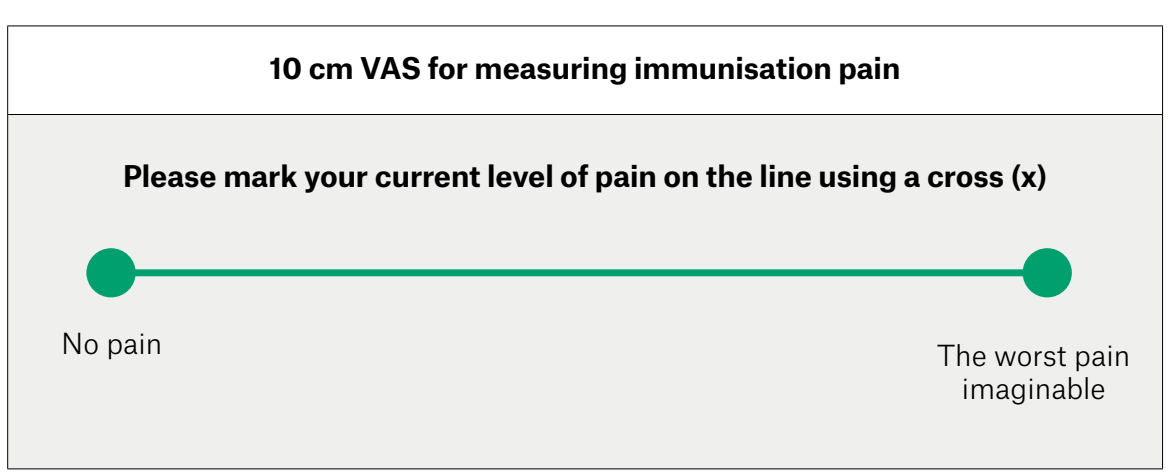

Figure 1. Self-report form for visual analogue scale (VAS) measurement 
(Figure 2). Additionally, the vaccination sites will be assessed for evidence of side effects if patients present opportunistically or for review because of side effects.

\section{Statistical analysis}

Statistical analysis will be performed using SPSS Statistics version 26 (IBM SPSS Statistics for Windows, Version 26.0. Armonk, NY: IBM Corp). Primarily, an intention-to-treat analysis of all randomised participants will be performed. Each individual will be considered as the unit of analysis. $P$ values will be two-tailed with $P<0.05$ considered statistically significant. Pain VAS pain scores between the ice and cold pack groups will be compared using an independent t-test or a Mann-Whitney U test as appropriate. Multiple linear and/ or logistic regression analysis (dependent on the baseline variable) will be conducted if baseline differences exist between participant groups and adjustment for confounders is required. Secondary outcomes will be analysed using two-tailed Fisher's exact probability testing. Sensitivity testing will be conducted for any participants lost to follow-up. Per-protocol analysis will evaluate potential effects of systematic biases due to non-compliers.

\section{Ethics}

This research will be guided by the National Health and Medical Research Council (NHMRC) national Statement on Ethical Conduct in Human Research, ${ }^{22}$ and the study protocol has been approved by the James Cook University Human Research Ethics Committee (approval number H7871).

It is not anticipated that the ice packs and cold packs will pose any significant risk of harm to participants. All potential participants will be provided with written advice of the protocols, benefits and risks of the study prior to obtaining informed consent. To prevent medical students being coerced to participate, an independent research officer will be assigned to oversee the recruitment and consent process. Individual respondent data will be de-identified, and all data and consent forms stored in a secured cupboard for the duration of the trial, then transferred to a locked safe at the conclusion of the study.

\section{Discussion}

Primary healthcare is a patient's first contact with health services and is likely the most accessed aspect of the Australian healthcare system. With current emphasis on the importance of high-quality primary healthcare in reducing the overall burden of disease, there is a greater need for clinical research focused on general practice. However, few RCTs are conducted in primary care settings. ${ }^{23}$ Researchers are often challenged with methodological difficulties as well as poor recruitment of patients and clinicians. ${ }^{24}$ These are further confounded by financial constraints. Primary care research in Australia receives limited funding, with only $1.9 \%$ of NHMRC grants awarded to general practice research between 2000 and $2008 .^{25}$

The research question is practical, clinically relevant and feasible to be investigated within a budget of $\$ 1000$. Ice packs are readily available in primary care settings and have a quick onset of analgesic action. ${ }^{11}$ The intervention is cost effective, $\$ 0.50$ per dose, and has little-to-no associated adverse effects. Therefore, the researchers believe that this

\begin{tabular}{|c|c|c|}
\hline \multicolumn{3}{|c|}{ Please report yes/no for the presence of any of the following } \\
\hline & Yes & No \\
\hline \multicolumn{3}{|c|}{ Redness at the injection site } \\
\hline \multicolumn{3}{|c|}{ Itching at the injection site } \\
\hline \multicolumn{3}{|c|}{ Pain at the injection site } \\
\hline \multicolumn{3}{|c|}{ Swelling at the injection site } \\
\hline \multicolumn{3}{|c|}{ Other skin changes - please specify } \\
\hline Anaphylaxis & & \\
\hline
\end{tabular}

Figure 2. Self-report form for potential adverse reactions (to be returned within 24 hours) will engage participating general practices and doctors, facilitate patient recruitment as well as increase the potential for translation into clinical practice.

The researchers plan to recruit medical students because of convenience in organising the study around the JCUMSA annual student influenza vaccination clinic and achieving a relatively homogenous study population. Medical students are considered to have higher health literacy, which may be associated with less pain intensity and anxiety regarding needlebased procedures. ${ }^{26}$ Consequently, medical students may report lower pain scores when compared with the general population. The researchers are aware that this may affect results to some degree, but hope to still be able to detect a measurable difference in pain scale scores between the intervention and control groups.

\section{Potential benefits if the problem could be solved}

The trial will provide guidance to general practitioners (GPs) regarding the use of ice for vaccination-related pain and will inform current clinical guidelines and healthcare worker education. The study will be conducted in a tropical rural setting and thus will have a unique patient demographic exposed to warmer and more humid weather conditions. Since the study 
protocol is pragmatically designed using a simple intervention that is accessible to GPs, the findings of this trial may be immediately translated into current clinical practice.

\section{Contingency plan}

In case of inadequate recruitment of patients, the researchers plan to open the invitation to medical students in non-clinical years. Feedback following immunisation is immediate, and thus high rates of follow-up are expected. Any students not followed up will be analysed using intention-totreat principles. The researchers have not planned to perform an interim analysis as the use of ice is a minor intervention, and the outcome of a surgical site infection is usually a minor medical issue that is treated with a course of antibiotics. An interim analysis is not feasible for this study, as it would considerably increase the required sample size.

\section{Dissemination}

The project is due for completion one year after commencement of data collection. The translation of important findings to clinical practice will be facilitated through conference presentations, medical journals and digital media. A written lay summary of the results will also be displayed at participating general practices and clinical schools for the information of study participants.

\section{Conclusion}

Timely clinical research is pivotal to ensure that GPs, the end-users of the research process, have access to evidence-based clinical guidelines and tools to optimise patient care. ${ }^{27}$ Overall, this trial has the potential to guide GPs regarding the use of ice packs to reduce injection-related pain, and to inform current clinical guidelines.

\section{Authors}

Yashodha Ediriweera MBBS (Hons), Resident Medical Officer, Royal Brisbane and Women's Hospital, Brisbane, QId

Jennifer Banks BBus, MBS, PhD, Senior Research Support, Mackay Clinical School, College of Medicine and Dentistry, James Cook University, Mackay, Qld Leanne M Hall PhD, Senior Research Officer, Mackay Clinical School, College of Medicine and Dentistry, James Cook University, Mackay, Qld
Clare Heal MBChB, DRANZCOG, DipGUMed, FRACGP, MPHTM, PhD, Promotional Chair, Discipline of General Practice and Rural Medicine, Mackay Clinical School, College of Medicine and Dentistry, James Cook University, Mackay, Qld

Competing interests: $\mathrm{CH}$ is a member of the Australian Journal of General Practice Editorial Advisory Committee.

Funding: None.

Provenance and peer review: Not commissioned, externally peer reviewed.

Correspondence to:

yashodha.ediriweera@my.jcu.edu.au

\section{Acknowledgements}

The research team would like to thank Dr Micheal Wohlfahrt, Dr Annaliese Wohlfarht, Mackay City Medical practice and Ms Mia Browning for their contributions to this study.

\section{References}

1. Mawhorter S, Daugherty L, Ford A, Hughes R, Metzger D, Easley K. Topical vapocoolant quickly and effectively reduces vaccine-associated pain: Results of a randomized, single-blinded, placebocontrolled study. J Travel Med 2004;11(5):267-72. doi: 10.2310/7060.2004.19101.

2. Australian Institute of Health and Welfare. The burden of vaccine preventable diseases in Australia. Canberra, ACT: AlHW, 2019. Available at www.aihw.gov.au/reports/immunisation/burdenof-vaccine-preventable-diseases-summary/ contents/burden-of-vaccine-preventable-diseases [Accessed 21 June 2020].

3. Nir Y, Paz A, Sabo E, Potasman I. Fear of injections in young adults: Prevalence and associations. Am J Trop Med Hyg 2003;68(3):341-44.

4. Bienvenu OJ, Eaton WW. The epidemiology of blood-injection-injury phobia. Psychol Med 1998;28(5):1129-36. doi: 10.1017/ s0033291798007144.

5. Gidudu JF, Walco GA, Taddio A, et al. Immunization site pain: Case definition and guidelines for collection, analysis, and presentation of immunization safety data. Vaccine 2012;30(30):4558-77. doi: 10.1016/j. vaccine.2012.03.085.

6. Melzack R, Wall PD. Pain mechanisms: A new theory. Science 1965;150(3699):971-79. doi: 10.1126/science.150.3699.971.

7. Kunesch E, Schmidt R, Nordin M, Wallin U, Hagbarth KE. Peripheral neural correlates of cutaneous anaesthesia induced by skin cooling in man. Acta Physiol Scand 1987;129(2):247-57. doi: 10.1111/j.1748-1716.1987.tb08065.x.

8. Schoenfeld AD, Lox CD, Chen CH, Lutherer LO. Pain threshold changes induced by acute exposure to altered ambient temperatures. Peptides 1985;6 Suppl 1:19-22. doi: 10.1016/0196-9781(85)90007-5

9. Breslin M, Lam P, Murrell GA. Acute effects of cold therapy on knee skin surface temperature: Gel pack versus ice bag. BMJ Open Sport Exerc Med 2015;1(1):e000037. doi: 10.1136/bmjsem-2015000037.

10. Cameron M. Physical agents in rehabilitation. 4th edn. St Louis, MI: Elsevier, 2017; pp. 129-44.

11. Akcimen M, Bedel C, Selvi F. Application of ice and vapocoolant spray to reduce tetanus vaccine pain: A prospective, randomized, controlled, clinical study. Ann Med Res 2019;26(6):995-98 doi: 10.5455/annalsmedres.2019.02.112.

12. Hall LM, Ediriweera $Y$, Banks J, Nambiar A, Heal C. Cooling to reduce the pain associated with vaccination: A systematic review. Vaccine 2020;38(51):8082-89. doi: 10.1016/j. vaccine.2020.11.005.

13. Taddio A, Lord A, Hogan ME, et al. A randomized controlled trial of analgesia during vaccination in adults. Vaccine 2010;28(32):5365-69. doi: 10.1016/j.vaccine.2010.05.015.

14. Standard Protocol Items: Recommendations for Interventional Trials (SPIRIT) Group. SPIRIT 2013 checklist: Recommended items to address in a clinical trial protocol and related documents. Toronto, CA: SPIRIT, 2020. Available at www.spirit-statement.org/wp-content/ uploads/2013/01/SPIRIT-Checklist-download8Jan13.pdf [Accessed 23 July 2020].

15. Sealed Envelope. 2021. Create a blocked randomisation list. London, UK: Sealed Envelope, 2020. Available at www.sealedenvelope.com/ simple-randomiser/v1/lists [Accessed 9 April 2020].

16. The Royal Australian College of General Practitioners. Guidelines for preventive activities in general practice. 9th edn. East Melbourne, Vic: RACGP, 2016.

17. National Aboriginal Community Controlled Health Organisation and the Royal Australian College of General Practitioners. Chapter 9: Respiratory health - Influenza prevention. In: National guide to a preventive health assessment for Aboriginal and Torres Strait Islander people. East Melbourne, Vic: RACGP, 2018.

18. Hawker GA, Mian S, Kendzerska T, French M. Measures of adult pain: Visual Analog Scale for Pain (VAS Pain), Numeric Rating Scale for Pain (NRS Pain), McGill Pain Questionnaire (MPQ), Short-Form McGill Pain Questionnaire (SF-MPQ), Chronic Pain Grade Scale (CPGS), Short Form-36 Bodily Pain Scale (SF-36 BPS), and Measure of Intermittent and Constant Osteoarthritis Pain (ICOAP). Arthritis Care Res (Hoboken) 2011;63 Suppl 11:S240-52. doi: 10.1002/acr.20543.

19. Nahm FS, Lee PB, Park SY, et al. Pain from intramuscular vaccine injection in adults. Rev Med Chil 2012;140(2):192-97. doi: 10.4067/S003498872012000200007.

20. Todd KH, Funk KG, Funk JP, Bonacci R. Clinical significance of reported changes in pain severity. Ann Emerg Med 1996;27(4):485-89. doi: 10.1016/ s0196-0644(96)70238-x.

21. Cohen M, Wolfe R, Mai T, Lewis D. A randomized, double blind, placebo controlled trial of a topical cream containing glucosamine sulfate, chondroitin sulfate, and camphor for osteoarthritis of the knee. J Rheumatol 2003;30(3):523-28.

22. National Health and Medical Research Council, the Australian Research Council, Universities Australia. National statement on ethical conduct in human research. Canberra, ACT: Commonwealth of Australia, 2018. Available at www.nhmrc.gov.au/ about-us/publications/national-statement-ethicalconduct-human-research-2007-updated-2018 [Accessed 23 July 2020].

23. Mendis K, Kidd MR, Schattner P, Canalese J. A bibliometric analysis of Australian general practice publications from 1980 to 2007 using PubMed. Inform Prim Care 2010;18(4):223-33. doi: 10.14236/jhi.v18i4.778.

24. Rendell JM, Merritt RD, Geddes JR. Incentives and disincentives to participation by clinicians in randomised controlled trials. Cochrane Database Syst Rev 2007;2007(2):MR000021. doi: 10.1002/14651858.MR000021.pub3.

25. Mclntyre EL, Mazza D, Harris NP. NHMRC funding for primary health care research, 
2000-2008. Med J Aust 2011;195(4):230. doi: 10.5694/j.1326-5377.2011.tb03292.x.

26. Köppen PJ, Dorner TE, Stein KV, Simon J, Crevenna R. Health literacy, pain intensity and pain perception in patients with chronic pain. Wien Klin Wochenschr 2018;130(1-2):23-30. doi: 10.1007/ s00508-017-1309-5.

27. Steel N, Abdelhamid A, Stokes T, et al. A review of clinical practice guidelines found that they were often based on evidence of uncertain

relevance to primary care patients. J Clin

Epidemiol 2014;67(11):1251-57. doi: 10.1016/j.

jclinepi.2014.05.020.

correspondence ajgp@racgp.org.au 